\title{
Ratio of Neutrophil/lymphocyte and Platelet/lymphocyte in Paediatric Patient with Cutaneous Leishmaniasis
}

B Sula ${ }^{1}, \mathrm{R}$ Tekin ${ }^{2}$, İ Yolbaş ${ }^{3}, \mathrm{~F} \mathrm{Aktar}^{3}, \mathrm{H} \mathrm{Uçak}^{1}$

\begin{abstract}
Background: In the present study, we aimed to detect the relation between Neutrophile/Lymphocyte, Platelet/Lymphocyte rates, platelet parameters and cutaneous leishmaniasis.

Materials and Methods: Eighty paediatric patients between One and 15 years of age who were admitted to Dermatology clinic of Dicle University and diagnosed with cutaneous leishmaniasis without any chronic systemic diseases were enrolled into the study. As a control group, 80 healthy children with age ranged between one and 16 years who admitted to paediatrics department for general health control and did not have any systemic disease known were included.
\end{abstract}

Results: Eighty patients admitted because of cutaneous leishmaniasis included 40 females (50\%) and 40 males $(50 \%)$; the age average was $8.53 \pm 3.92$. In the control group, 80 patients consisted of 34 (42.5\%) males and $46(57.5 \%)$ females. No statistically significant difference was detected in age, lymphocyte, neutrophile/lymphocyte rate and platelet values when compared with the control group whereas white blood cell count, neutrophile count, platelet/Lymphocyte rate and mean platelet volume values were found significantly lower in the patent group than the control group. Platelet distribution width was detected statistically significant higher in the patient group when compared with the control group.

Conclusion: Since haemogram parameters such as platelet/lymphocyte rate, white blood cell count, neutrophile count, mean erythrocyte volume, mean platelet volume and platelet distribution width maybe used to determine inflammatory status and to assess treatment efficiency for the patients with cutaneous leishmaniasis. We believe that to evaluate all these with other inflammatory markers might reveal more accurate results.

Keywords: Childhood, cutaneous leishmaniasis, lymphocyte rate, neutrophile, platelet

From: ${ }^{1}$ Dicle University Medical School, Department of Dermatology, Diyarbakır, Turkey, ${ }^{2}$ Dicle University Medical School, Department of Infectious Diseases and Clinical Microbiology, Diyarbakır, Turkey and ${ }^{3}$ Dicle University Medical School, Department of Pediatrics, Diyarbakir, Turkey.

Correspondence: Dr B Sula, Dicle University Medical School, Department of Dermatology, Diyarbakir, Turkey. Turkey, E-mail: bulentsula@ hotmail.com, Fax: +90 4122488523 


\section{INTRODUCTION}

Cutaneous leishmaniasis is an intracellular infectious disease which is caused by leishmania protozoon parasites in leishmania genus and transmitted by a sandfly vector. Parasites enter into the body through the skin and infect reticuloendothelial system cells and finally cause clinical manifestations such as cutaneous, mucocutaneous and visceral leishmaniasis. The most common form is cutaneous lesihmaniasis. The disease starts with an erythamatous papule clinically and then a nodule with a diameter of 1 to $2 \mathrm{~cm}$ appears within a couple of months. An ulcer covered by a crust appears on the center of this nodule. Lesions generally meliorate by leaving a depressed scar within 1 to 2 years (Figure 1). Laboratory parameters may change in cutaneous leishmaniasis. White Blood Cell Count (WBC), neutrophile, lymphocyte and platelet counts, mean platelet volume (MPV) and platelet distribution width (PDW) parameters are laboratory markers which are easily and routinely measured in haemogram parameters and used for many cases such as evaluation of a systemic inflammation, diagnosis and treatment of infectious diseases. Recent studies reported that some changes appear in WBC, neutrophile, lymphocyte and platelet counts, MPV, PDW, neutrophile/lymphocyte (NLR) and Platelet/lymphocyte rates (PLR) and these are related with severity and prognosis of the diseases (2-4).

As of our knowledge and research, there is not any study on MPV, PDW, NLR and TLR for cutaneous leishmaniasis. In the present study, we aimed to detect possible changes in WBC, neutrophile, lymphocyte and platelet counts, MPV, PDW, NLR and PLR rates for cutaneous lesihmaniasis which is an infectious disease. 


\section{SUBJECTS AND METHODS}

Eighty paediatric patients with age range of one and 15 years who were admitted to Dermatology clinic of Dicle University and treated after diagnosis of cutaneous leishmaniasis after clinical and laboratory examination without any concomitant chronic systemic disease between January, 2011 and November, 2014 were enrolled into the present study. In our clinic, patients with lesions which may create chronic and cosmetic problems, patients with inflammatory lesions larger than $2-5 \mathrm{~cm}$, lesions on patients with immunosuppressive patients, patients with facial lesions such as lesions on the eyelid, ear, nose and lips which may cause dysfunction and deformity, those with mucosal involvement or patients with cutaneous lesihmaniasis who have multiple ( 5 or more) lesions are admitted for treatment and followed up.

As a control group, 80 healthy children with age ranged between one and 16 years who admitted to paediatrics department for general health control and did not have any systemic disease known were included. For automated blood count, samples were collected from antecubital vein into vacuum tubes with EDTA (15\% K3 EDTA $0.054 \mathrm{ml} / 4.5 \mathrm{~mL}$ blood) and samples collected were analysed within one hour. Complete blood count measurements were performed with an automated complete blood count device (A Sysmex XE-2100). Ages, genders and WBC, platelet count, PDW, MPV, mean corpuscular volume (MCV), neutrophile and lymphocyte values among pre-treatment complete blood count parameters were recorded at admission. NLR and PLR were calculated and compared between the groups.

SPSS 15.0 program (SPSS for Windows 15, Chicago, Illinois, USA) was used for statistical analysis of data. Continuous variables were determined as mean \pm Standard deviation; categorical variables were determined as (\%). Ki-square test and Student $t$-test were 
used for categorical and continuous variables. Statistically significance was accepted as $p<$ 0.05 .

\section{RESULTS}

Eighty patients with age range of one and 15 years admitted because of cutaneous leishmaniasis included 40 females $(50 \%)$ and 40 males $(50 \%)$; the age average was $8.53 \pm$ 3.92. There were 80 cases with age range of one and 16 years in the control group including $34(42.5 \%)$ males and $46(57.5 \%)$ females; the age average was $8.50 \pm 3.91$. Patients were divided into three groups according to their ages. There were 20 patients $(25 \%)$ whose age was under five years, 31 patients $(38.8 \%)$ whose age was between six and 10 years and 29 patients $(36.3 \%)$ with age range of 11 and 15 years.

No statistically significant difference was observed in age, lymphocyte, NLR and Platelet count in the patient group when compared with the control group whereas WBC, neutrophile count, MCV, PLR rate and MPV values were found significantly lower in the patient group than the control group. PDW was detected statistically and significantly higher in the patient group when compared with the control group (Table I).

Table I. Evaluation of haemogram parameters in the patient and control groups.

\begin{tabular}{llll}
\hline Parameters & $\begin{array}{l}\text { Patient } \\
(\text { Mean } \pm \mathrm{SD})\end{array}$ & $\begin{array}{l}\text { Control } \\
(\text { Mean } \pm \mathrm{SD})\end{array}$ & $\boldsymbol{p}$ \\
\hline Age (year) & $8.53 \pm 3.92$ & $8.50 \pm 3.91$ & 0.952 \\
WBC (K/uL) & 8306.50 & 9170.62 & $\mathbf{0 . 0 2 9}$ \\
Neutrophil (K/uL) & 3805.87 & 5095.25 & $\mathbf{0 . 0 0 0}$ \\
Lymphocyte (K/uL) & 3325.37 & 3133.00 & 0.418 \\
Platelets (K/uL) & 321162.50 & 347587.50 & 0.083 \\
NLR & $2.02 \pm 3.21$ & $1.80 \pm 1.01$ & 0.563 \\
MCV (fL) & 78.98 & 83.25 & $\mathbf{0 . 0 0 0}$ \\
MPV (fL) & 7.32 & 8.15 & $\mathbf{0 . 0 0 0}$ \\
PDW (\%) & 17.10 & 13.37 & $\mathbf{0 . 0 0 0}$ \\
PLR & $122.30 \pm 48.22$ & $152.70 \pm 227.31$ & $\mathbf{0 . 0 1 4}$ \\
\hline
\end{tabular}

NLR: Neutrophil / Lymphocyte Ratio, PLR: Platelet/Lymphocyte Ratio, SD: Standard Deviation 


\section{DISCUSSION}

Cutaneous leishmaniasis continues to be an important health problem in many countries of the world as well as our country. The disease is frequently observed in Southeast Anatolia region and Çukurova region endemically. Leishmaniasis presents a clinical spectrum in form of localized (cutaneous leishmaniasis) or disseminated infection (cutaneous, mucosal or visceral) (1). Clinical progress of the disease depends on the balance between activation of the cytokines which are majorly produced by Th-1 cells and deactivation of the cytokines produced by subunits of other regulatory T-cells (5). Furthermore, neutrophiles are the first responding cells within first 30 minutes following sandfly bite and they phagocyte the parasites. Immune response of the neutrophiles against leishmania is regulated through a direct interaction by cytokines, chemokines, nature of the granular content and settled inflammatory cells in the infection area. Decrease in acute neutrophile infiltration causes increase in host defense against the parasite $(6,7)$. Haematological disorders such as anemia, neutropenia, thrombocytopenia and pancytopenia may be observed in systemic involvement of leishmaniasis. Many mechanisms were blamed as a cause for this. In the studies conducted, neutropenia development was detected due to destruction in the spleen and changes appeared in polymorphonuclear leukocytes (PNL). Significant decrease in PNL reserve in the bone marrow was shown in some patients. Furthermore, it was reported that rapid destruction of antibody-coated cells (membrane-associated antiplatelet, antineutrophilic and antierythrocytic IgG antibodies) caused pancytopenia $(1,8)$. Parasite load, enlargement of the spleen size and disease period are generally associated with severity of pancytopenia. It was shown in the studies that amastigotes exist in macrophages, PNL and eosinophilles. It was also suggested that anemia is caused by phagocytosis of immature erythroblasts and ineffective erythropoesis due to macrophage hyperactivity in the bone marrow $(8,9)$. 
Systemic inflammatory status may be measured through different markers today. Use of NLR as a marker for different diseases such as diabetes mellitus, atherosclerosis, hypertension, metabolic syndrome, myocardial infarction, cancers of the lung, ovaries and stomach as an indicator for systemic inflammatory status $(2,10)$.

In the study conducted by Sen et al, higher neutrophile count, NLR and lower lymphocyte count were detected in the patients with psoriasis than the control group. They suggest that this may be used as a simple, cost-efficient and practical marker to evaluation the systemic inflammation for psoriasis (10) Ahsen et al, found NLR significantly higher in patients with familial mediterranean fever (FMF) when compared with the control group in their study and they reported that such marker may be used as an indicator for subclinic inflammation for the patients with FMF (11). In a study conducted on 92 patients diagnosed with community-acquired bacteremia, higher CRP, NLR and lower lymphocyte values were observed in patients with positive blood culture than the patients with negative blood culture whereas no difference was detected in WBC and neutrophile values. Consequently, they suggested that lymphocytopenia and NLR would be a better marker than routine parameters such as WBC, CRP and neutrophile count (12). There is not any scientific study on NLR in the patients with cutaneous leishmaniasis in the literature research. In the present study, NLR was detected higher in the patients with leishmaniasis than the control group even it is not significant. Lower WBC and neutrophile count were statistically significant than the control group and no statistically significant difference was found in lymphocyte values between the two groups.

Some parameters which may be useful for diagnosis of various platelet diseases are also analysed in haemogram. These parameters are especially helpful for differential diagnosis of thrombocytopenia and thrombocytosis. It was reported in the previous studies that increased MPV reflects the inflammatory load and treatment response for different 
diseases and it is closely correlated with inflammation (13). Studies showed that MPV increases in cardiovascular diseases such as myocardial infarction, stroke and peripheral arterial disease; however, it may increase or decrease in rheumatic diseases. PDW is a quantitative marker for platelet volume which is useful for differentiation of reactive thrombocytosis and essential thrombocytemia (14, 15). Association of TLR with bad prognosis was reported for different cancer types and cardiovascular diseases $(16,17)$. In the study conducted by Bozkurt et al, MPV and RDW were analysed before and after the treatment in patients with Brucellosis. MPV and RDW were detected lower in the pretreatment group when compared with the post-treatment group. Furthermore, a statistically significant difference was found on ESR, MPV, RDW, haemoglobin, CRP, PLT and WBC between the groups (4). In a study performed in Iran, higher platelet and lower MPV levels were detected in 100 paediatric patients with inflammatory and infectious diseases during active disease term when compared with the recovery period. They suggested that these markers may be used as a reliable marker to show activity of the disease and to assess the treatment response (3). In the study of Boyraz et al for TLR on 105 patients with ankylosing spondylotis, no significant difference was observed on NLR rate whereas significantly lower values were reported on TLR than the control group (16). The relation between severity of the coronary artery disease and TLR was investigated in another study that TLR was found significantly higher in the patients with severe coronary artery disease when compared with the control group and patients with mild-intermediate coronary artery disease. There was not any scientific study conducted on MPV, RDW and TLR in patients with cutaneous leishmaniasis which is a parasitary infection in the literature. In the present study, statistically significant lower MPV and higher RDW values were observed in the patient group than the control group; there was not any statistically significant difference was found in platelet count. Furthermore, TLR were compared and they were found lower in terms of statistical 
significance in the patients with leishmaniasis than the control group. Results of the present study was generally consistent with previous studies conducted for different infectious and inflammatory diseases.

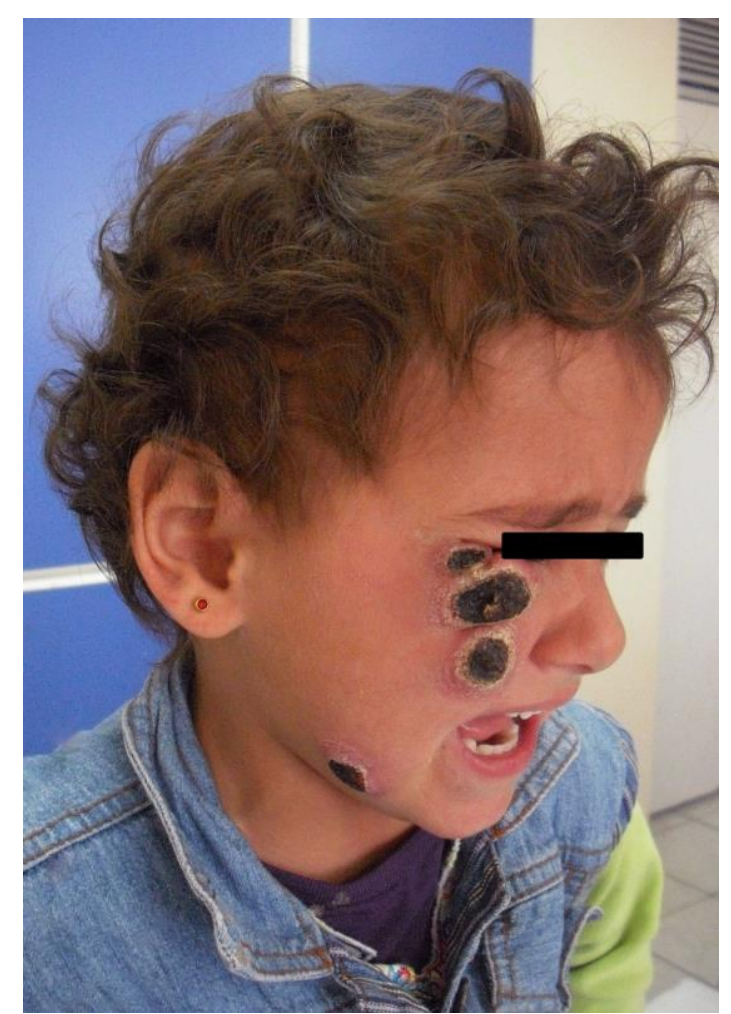

Figure 1. Erythematous, ulcerated nodular lesions covered with black crusts.

\section{CONCLUSION}

Parameters such as TLR, WBC, neutrophile count, MCV, PDW and MPV which may be easily measured in a routine haemogram test may be used to determine the inflammatory status and to assess the treatment efficiency in patients with cutaneous leishmaniasis; evaluation of these parameters with other inflammatory markers would reveal more accurate results. We believe that more and comprehensive studies are required to show efficiency of these parameters clearly.

Disclosures: Financial disclosure statements have been obtained, and no conflicts of interest have been reported by the authors or by any individuals in control of the content of this article.

No conflicts of interest to declare 


\section{REFERENCES}

1. Uzun S: Leishmaniasis. Dermatoloji. Ed. Tüzün Y, Gürer MA, Serdaroğlu S, Oğuz O, Aksungur VL. 3. Ed. İstanbul, Nobel medicine bookstore, 2008;659-682.

2. Zahorec R. Ratio of neutrophil to lymphocyte counts-rapid and simple parameter of systemic inflammation and stress in critically ill. Bratisl Lek Listy 2001; 102: 5-14.

3. Zareifar S, Farahmand Far MR, Golfeshan F, Cohan N. Changes in platelet count and mean platelet volume during infectious and inflammatory disease and their correlation with ESR and CRP. J Clin Lab Anal 2014; 28 (3): 245-248.

4. Bozkurt F, Aslan E, Deveci Ö, Tekin R. Evaluatıon of Mean Platelet Volume Levels in Patients With Brucellosis. Anatol J Clin Investig 2014; 8 (3): 126-129.

5. Ribeiro-Gomes FL, Peters NC, Debrabant A, Sacks DL. Efficient capture of infected neutrophils by dendritic cells in the skin inhibits the early anti-leishmania response. PLoS Pathog 2012; 8 (2): e1002536.

6. McConville MJ, Naderer T. Metabolic pathways required for the intracellular survival of Leishmania. Annu Rev Microbiol 2011; 65: 543-561.

7. Ribeiro-Gomes FL, Sacks D. The influence of early neutrophil-Leishmania interactions on the host immune response to infection. Front Cell Infect Microbiol 2012; $2: 59$.

8. Rai ME, Muhammad Z, Sarwar J, Qureshi AM. Haematological findings in relation to clinical findings of visceral Leishmaniasis in Hazara Division. J Ayub Med Coll Abbottabad 2008; 20: 40-43.

9. Marwaha N, Sarode R, Gupta RK, et al. Clinico-hematological characteristics in patients with kala azar. A study from north-west India. Trop Geogr Med 1991; 43: 357-362. 
10. Sen BB, Rifaioglu EN, Ekiz O, Inan MU, Sen T, Sen N. Neutrophil to lymphocyte ratio as a measure of systemic inflammation in psoriasis. Cutan Ocul Toxicol 2014; 33 (3): 223-227.

11. Ahsen A, Ulu MS, Yuksel S, Demir K, Uysal M, Erdogan M, Acarturk G. As a new inflammatory marker for familial Mediterranean fever: neutrophil-to-lymphocyte ratio. Inflammation 2013; 36 (6): 1357-1362.

12. de Jager CP, van Wijk PT, Mathoera RB, de Jongh-Leuvenink J, van der Poll T, Wever PC. Lymphocytopenia and neutrophil-lymphocyte count ratio predict bacteremia better than conventional infection markers in an emergency care unit. Crit Care 2010; 14 (5): R192.

13. Akarsu S, Kurt ANÇ, Kurt A, Varol İ, Şen Y. Thrombocyte volume parameters in different disease groups. Turkish Pediatrics Archive 2006; 41: 208- 213.

14. Yazici S, Yazici M, Erer B, et al. The platelet indices in patients with rheumatoid arthritis: mean platelet volume reflects disease activity. Platelets 2010; 21: 122-125.

15. Wiwanitkit V. Plateletcrit, mean platelet volume, platelet distribution width: its expected values and correlation with parallel red blood cell parameters. Clin Appl Thromb Hemost 2004; 10 (2): 175-178.

16. Boyraz İ, Koç B, Boyacı A, Tutoğlu A, Sarman H, Özkan H. Ratio of neutrophil/lymphocyte and platelet/lymphocyte in patient with ankylosing spondylitis that are treating with anti-TNF. Int J Clin Exp Med 2014; 7 (9): 2912-2915.

17. Yüksel M, Yıldız A, Oylumlu M ve ark. The association between platelet/lymphocyte ratio and coronary artery disease severity. Anadolu Kardiyol Derg 2014; 14 (0): 000000, DOI:10.5152/akd.2014.5565. 